\title{
Regression of established subcutaneous B16-F10 murine melanoma tumors after gef gene therapy associated with the mitochondrial apoptotic pathway
}

\author{
Jose Prados ${ }^{1,2^{*}}$, Consolación Melguizo ${ }^{1,2^{*}}$, Raúl Ortiz ${ }^{1,2}$, Houria Boulaiz ${ }^{1,2}$, Esmeralda Carrillo ${ }^{1,2}$, \\ Ana Segura ${ }^{3}$, Jose Juan Rodríguez-Herva ${ }^{3}$, Juan Luis Ramos ${ }^{3}$ and Antonia Aránega ${ }^{1,2}$ \\ 'Institute of Biopathology and Regenerative Medicine (IBIMER), Granada, Spain; \\ 2Department of Human Anatomy and Embryology, School of Medicine, Lniversity of Granada, Granada, Spain; \\ ${ }^{3}$ CSIC-Estación Experimental del Zaidín, Department of Environmental Protection, Granada, Spain \\ Correspondence. Dr Jose Prados, Instituto de Biopatologia y Medicina Regenerativa (IBIMER), Depto. de Anatomía y Enbriología, Facultad de \\ Medicina, Universidad de Granada, E-18071 Granada, Spain, Tel.+\$34 958 242068, Fax: +34 958 246296, e-mail: icprados@ugr.es \\ *These authors contributed equally to this work.
}

\begin{abstract}
Novel treatment modalities, including gene therapy, are needed for patients with advanced melanoma. We evaluated whether the gef gene, a suicide gene from Escherithia coli, had a significant cytotoxic impact on melanoma in vivo. First, we used a non-viral gene delivery approach (pcDNA3.1 $/ \mathrm{gef}$ ) to study the inhibition of melanoma cells (B16-F10) proliferation in vitro. Secondly, we used direct intra-tumoral injection of pcDNA3.1/gef complexed with jetPEI to deliver gef cDNA to rapidly growing murine melanomas. We demonstrated that gef gene not only has an antiproliferative effect on Bl6-F10 cells in vitro, but also
\end{abstract}

\section{Introduction}

Melanoma represents only $4 \%$ of all skin cancers, but nearly $80 \%$ of skin cancer deaths, predominantly because of metastatic spread (1). Apart from surgery, treatment options for melanoma, particularly metastatic melanoma, are relatively limited. As melanoma is a highly therapy-refractory tumor, it demands effective therapies combinations (2). Suicide gene therapy has been proposed as a strategy for the treatment of intractable cancers and has been assayed in some clinical trials by itself or in combination with other therapies (tumor irradiation or chemotherapy). In melanoma, strategies to facilitate apoptosis by gene therapy may be an alternative or complementary strategy for its treatment (3) as it has been demonstrated that apoptosis deficiency is a critical factor for therapy resistance in this tumor (4).

Classical cancer suicide gene therapy employs genes encoding enzymes that convert non-toxic prodrugs into cylotoxic compounds (5). However, these prodrug systems have been assayed in melanoma both in vitro and in vivo with limited induces an important decrease in melanoma tumor volume (77.7\% in 8 days) in vivo. Interestingly, after gef gene treatment, melanoma showed apoptosis activation associated with the mitochondrial pathway, suggesting that the induction of this death mechanism may be an effective strategy for its treatment. Our in vivo results indicate that gef gene might become a suitable therapeutic strategy for patients with advanced melanoma.

Key words: apoptosis - caspase - gef gene - melanoma mitochondria

results $(6,7)$. As an attractive alternative to this strategy, therapeutic genes that directly encode cytotoxic proteins could be used. In contrast to classical suicide genes that act by disrupting DNA synthesis (targeting only rapidly dividing cells) these new toxins may act killing both quiescent and rapidly dividing tumor cells and may be effective for aggressively growing tumors as well as for those that grow more slowly. Many genes encoding cylotoxic products have been evaluated as gene therapy approaches $(8,9)$. The most recent experiences with genes expressing toxins from bacteria $(10)$, from plants (11) or from bacteriophage (12) showed a high cylotoxic impact on tumoral cells derived from different tissues.

In this context, our group has developed last year a direct cancer gene therapy system based in the suicide gene named gef. The gef gene, member of a gene family with homologous cell-killing functions, encodes a membrane protein of 50 amino acids which is anchored in the cyloplasmic membrane by the $\mathrm{N}$-terminal portion, whereas the C-terminal part is located in the periplasm (13). Although activation of this protein induces arrest of respiration and 
death in bacterial cells the mechanism of action in tumoral cells is unclear. We have previously demonstrated that gef protein is able to induce changes in proliferation rate and differentiation degree of tumoral cells without having to use prodrugs $(14,15)$. However, its possible in vivo application could not be demonstrated yet.

In this study, we have evaluated for the first time the potential use of the gef gene for the treatment of melanoma tumors in vivo. Moreover, based on the knowledge that the gef gene encodes a cytotoxic protein that binds to cell membranes, we analysed gef's mechanism of action. We used the B16-F10 murine melanoma model because of its highly invasive and metastatic nature and the cationic lipids jetPEI to deliver gef cDNA to rapidly growing murine melanomas. Results obtained suggest that treatment with the gef gene significantly decreases tumor growh, inducing apoptosis in melanoma tumor cells by means of the mitochondrial pathway.

\section{Methods}

\section{Cells and reagents}

The B16-F10 murine melanoma cell line (CRL6475) was obtained from American Type Culture Collection and was grown in Dulbecco's modified eagle's medium (Sigma, St Louis, MO, USA), supplemented with $10 \%$ heat-inactivated foetal bovine serum, $2 \mathrm{~mm}$ L-glutamine, $40 \mathrm{mg} / 1$ gentamicin and $500 \mathrm{mg} / 1$ ampicillin (Antibióticos S.A, Madrid, Spain). Cells were maintained in monolayer culture at $37^{\circ} \mathrm{C}$ in an atmosphere containing $5 \% \mathrm{CO}_{2}$.

\section{gef transfection in B16-F10 cells}

The gef gene was amplified using specific primers (sense 5'-ATGAAGCAGCATAAGGCGATG-3' and antisense 5'TTACTCGGATTCGTAAGCCGTC-3') under the following conditions: $94^{\circ} \mathrm{C}$ for $1 \mathrm{~min}, 35$ cycles at $94^{\circ} \mathrm{C}$ for $1 \mathrm{~min}$, $53^{\circ} \mathrm{C}$ for $30 \mathrm{~s}$ and $72^{\circ} \mathrm{C}$ for $30 \mathrm{~s}$ and $72^{\circ} \mathrm{C}$ for $10 \mathrm{~min}$ and was subcloned into the pcDNA3.1-TOPO vector (Invitrogen, Barcelona, Spain) following the manufacturers' instructions. The resulting plasmid pcDNA3.1/gef was transformed into the subcloning efficiency $\mathrm{DH} 5$ alpha chemically competent Escherichia coli (Invitrogen). The correct DNA sequence was confirmed by sequence analysis using the T7 primer. One day before transfection, confluent cells were seeded into six-well plates $\left(2 \times 10^{5}\right.$ cells $/$ well). Briefly, a transfection mixture was prepared by adding $94 \mu \mathrm{l}$ of the serum-free medium and $6 \mu \mathrm{l}$ FuGENE- 6 reagent (Roche Diagnostic, Barcelona, Spain). After 5 min of incubation at room temperature, $2 \mu \mathrm{g}$ of plasmid DNA (pcDNA3.1/gef) were added (ratio 1:3). B16-F10 cells, yielding approximately $70 \%$ confluence, were transfected with gef gene-containing pcDNA3.1 vector. Cells were cultivated for $8 \mathrm{~h}$ at $37^{\circ} \mathrm{C}$, and the medium containing transfection mixture was then replaced with the growth medium.
A pcDNA3.1 plasmid in which the gef gene was absent was used as a negative control. The pcDNA3.1/green fluorescent protein (GFP) (provided by Dr. G. Ortiz) was used to optimize transfections conditions.

\section{Proliferation assays}

Parental and transfected cells growing in well plates were trypsinized after 24, 48, 72 and $96 \mathrm{~h}$ and collected. Cells were fixed and stained with $0.4 \%$ sulphorhodamine $\mathrm{B} / 1 \%$ acetic acid. Cells previously washed with $0.1 \%$ acetic acid were left in $10 \mathrm{~mm}$ Trizma for $15 \mathrm{~min}$ at room temperature. Optical density was then determined using a Titertek multiscan (Flow, Irvine, CA, USA) colorimeter at $492 \mathrm{~nm}$. Linearity of the SRB assay with cell number was tested for each B16-F10 cell stock before each cell growh experiment. B16-F10 cells transfected with empty vector were used in the proliferation assay as controls.

\section{In vitro and in vivo expression of gef gene}

Upregulation of mRNA expression of gef cDNA was determined by RT-PCR. RNA was extracted from transfected and parental cells with the RNeasy Mini kit (Qiagen, Valencia, CA, USA). RNA from tumor was obtained with the RNeasy Fibrous Tissue Mini Kit (Qiagen). cDNA was generated by means of the Promega reverse transcription system using total cellular RNA $(1 \mu \mathrm{g})$. PCR amplification of gef gene took place under the above-described conditions. RNA integrity was assessed by amplification of $\beta$-actin mRNA. Images were scanned and analysed using a Bio-Rad documentation system (Quantity One Analysis Software, Bio-Rad Laboratories, Barcelona, Spain). Relative gef mRNA expression was calculated as the ratio of gef to $\beta$-actin.

\section{Annexin $\mathrm{V}$ and propidium iodide staining}

Parental and transfected cells were washed twice with phosphate-buffered saline (PBS) and incubated in binding buffer containing annexin V-FITC $(25 \mu \mathrm{g} / \mathrm{ml})$ and propidium iodide $(25 \mu \mathrm{g} / \mathrm{ml})$ in the dark for $15 \mathrm{~min}$ at room temperature (Annexin V-FITC Apoptosis Detection Kit I; BD Pharmingen, San Diego, CA, USA). Then, binding buffer $(500 \mu \mathrm{l})$ was added and cells were immediately processed with a FACScan flow cytometer. Microscopy analysis was carried out by Technical Services from the Granada University in a Leica DMI6000 (Heidelberg, Germany) confocal microscope with laser Argon/Krypton.

\section{Assay for cytoplasmic mono- and oligonucleosomes}

The Cell Death Detection ELISA Kit (Boehringer, Mannheim, Germany) was used for assessing apoptosis in transfected cells following the manufacturer's protocol. Parental and transfected cells $\left(2 \times 10^{4}\right)$ were lysed and the cell lysates were overlaid and incubated in microtitre plate 
modules coated with antihistone antibody. Samples were then incubated with anti-DNA peroxidase followed by colour development with ABTS (2,2' -Azino-bis(3-Ethylbenzthiazoline-6-Sulfonic Acid)) substrate. Samples absorbance was determined with Titertek multiscan at $405 \mathrm{~nm}$.

\section{Measurement of mitochondrial membrane potential}

Parental and transfected cells were washed twice with cold PBS and incubated with $40 \mathrm{nM}$ DiOC6(3) for $15 \mathrm{~min}$ at $37^{\circ} \mathrm{C}$. Then, cells were washed with ice-cold PBS and resuspended in $500 \mathrm{ml}$ of PBS. Fluorescence intensities of DiOC6(3) were analysed on a FACScan (Becton Dickinson, San Jose, CA, USA) flow cytometer with excitation and emission settings of 484 and $500 \mathrm{~nm}$, respectively.

\section{Caspase activity assay}

Caspase- 9 and -8 activities were measured using caspase colorimetric assay kits (R\&D Systems, Minneapolis, MN, USA). Briefly, parental and transfected cells were washed twice with cold PBS and resuspended in $50 \mu$ of cold lysis buffer, incubated for $10 \mathrm{~min}$ and centrifuged for $1 \mathrm{~min}$ at $10000 \mathrm{~g}$ to precipitate cellular debris. Assay was performed in triplicate on a 96-well plate following the manufacturers' protocol. Results are expressed as the fold increase in pcDNA3.1/gef treated cells over that of control cells. Etoposide (Sigma, St. Louis, MO, USA) $(50 \mu \mathrm{mol} / \mathrm{l})$ was used as positive control of caspase activities in B16-F10 cells.

\section{Tumor induction and measurement}

For in vivo study, female C57BL/6 mice (Scientific Instrumentation Centre, Granada University, Granada, Spain) were used. All mice (weighing $25-30 \mathrm{~g}$ ) were maintained in a laminar air-flow cabinet at a room kept at $37^{\circ} \mathrm{C}$ temperature and $40-70 \%$ humidity with a 12 -h light/dark cycle under specific pathogen-free conditions. All studies on animal models were approved by the Ethical Committee of the Medical School of Granada University and performed according to its guidelines. Tumors were induced by subcutaneous injection of $5 \times 10^{5} \mathrm{~B} 16-\mathrm{F} 10$ cells into the left flanks of C57BL/6 mice. Tumors were allowed to grow to the appropriate size $\left(75 \mathrm{~mm}^{3}\right)$ before treatment (ideal minimal size for intratumoral injection). After reaching this volume (treatment day 0 ), tumors were measured at periodic intervals following treatment using a digital caliper by measuring the longest diameter (a) and the next longest diameter (b) perpendicular to (a). Using these measurements the tumor volume was calculated by the formula $V=a b^{2} \times \pi / 6$.

\section{Intra-tumoral plasmid treatment}

In vivo JetPEI (Polyplus-transfection Inc., New York, NY, USA) was used as a transfection enhancer reagent. PEV/DNA complexes with a ratio of 1:6 were prepared in a solution of
$10 \% \mathrm{w} / \mathrm{v}$ glucose. This was carried out in a two-step procedure for the preparation of a standard quantity of $20 \mathrm{Hg}$ of PEV/DNA complex, according to the manufacturer's instructions. Tumors were then treated intra-tumorally with pcDNA3.1/gef plasmid. The pcDNA3.1 LacZ plasmid was used to normalize transfection efficiency. Moreover, control groups (without treatment and treated with empty vector) were included. Treatments were administered during 14 days. Comparative study between treated and non-treated groups was realized during the first 8 days because of the high rate of mortality in control group.

\section{Histological analysis}

Tumors were fixed in $4 \%$ paraformaldehyde in PBS, embedded in paraffin and cut into 3-5 $\mu \mathrm{m}$ sections. Cells were immunofluorescently labelled with primary antitubulin mouse monoclonal antibody (1:500) (Sigma) followed by Texas Red dye-conjugated affinitPure Goat Anti-Mouse IgG + IgM (1:500) (Jackson ImmnoResearch Laboratories, West Grove, PA, USA). The presence of apoptotic cells within the tumor sections was evaluated by the TUNEL technique using the In Situ Cell Death Detection Kit Fluorescein (Roche, Mannheim, Germany) according to manufacturers' recommendations. Cell nuclei were counterstained with DAPI. Per cent apoptosis (apoptotic index) was determined by counting the number of apoptotic cells and dividing by the total number of cells in the field ( 5 high power fields/slide). Immunohistochemical analyses of caspases were realized using antiactive forms of caspase-9 (1:50) (Cell Signaling Technology, Inc, Danvers, MA, USA) and caspase-8 (1:100) (Imgenex, San Diego, CA, USA). FITC-conjugated antirabbit secondary antibody at room temperature for $1 \mathrm{~h}$ was used for the detection. Cell nuclei of cultures were counterstained with DAPI. Fluorescence images were captured using an Olympus DP11 microscope with a Nikon Eclipse Ti (Nikon Instruments Inc., New York, NY, USA) digital imaging system.

\section{Transmission electron microscopy}

Melanoma tumors grown in mice were collected, cut up into small pieces and immediately fixed with $2.5 \%$ glutaraldehyde in $0.1 \mathrm{M}$ cacodylate buffer $(\mathrm{pH} 7.2$ ) at room temperature for $1 \mathrm{~h}$. After postfixation with $1 \% \mathrm{OsO}_{4}$ in cacodylate buffer (room temperature, $2 \mathrm{~h}$ ), sections were dehydrated through graded ethanol concentrations with a final propylene oxide dehydration. Samples were then embedded in Epon 812 resin. Ultrathin sections, were stained with uranyl acetate and lead citrate and examined in a Hitachi H7000 transmission electron microscope (TEM, Hitachi High-Technologies Corporation, Tokyo, Japan).

\section{Statistical analysis}

SPSS 14 software (SPSS Inc, Chicago, IL, USA) was used for all statistical analyses. Results were compared by using 
the Student's $t$-test. All data are expressed as mean \pm SD. Differences were considered statistically significant at a $P$-value of $<0.05$.

\section{Results}

\section{Expression of gef gene and inhibition of cell growth in B16-F10 cells in culture}

In vitro evaluation of gef gene expression was performed by RT-PCR. As shown in Fig. 1a, an amplification fragment of

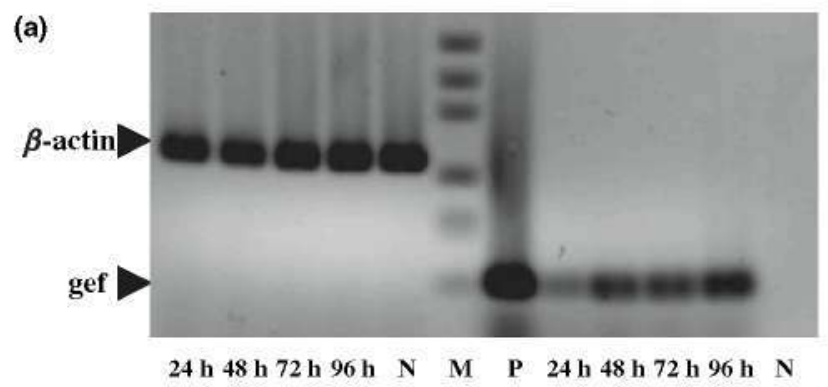

(b)
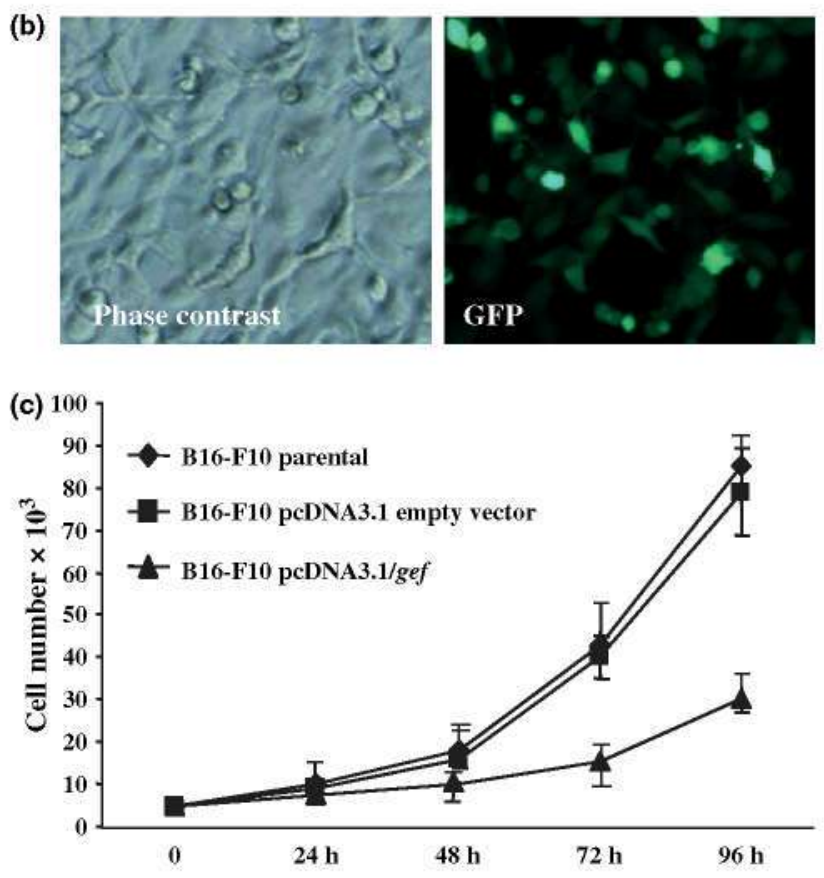

Figure 1. gef gene expression and growth rate inhibition in B16-F10 cells. (a) RT-PCR showing gef gene expression in B16-F10 transfected cells at different time periods. The integrity of the RNA was demonstrated using $\beta$-actin primers. N, negative control (B16-F10 nontransfected cells); $M$, molecular weight; $P$, positive control (pcDNA3.1/gef). (b) Representative photomicrograph (phase contrast and fluorescent images) of B16-F10 cells transfected with pCDNA3.1/GFP to optimize transfections conditions. (c) Growth of B16F10 cells expressing gef was detected by sulphorhodamine B assay. Cells transfected with pcDNA3.1/gef showed a clearly decreased growth rate compared to the control cells and cells transfected with pCDNA3.1 empty vector $(P<0.05)$. Data represent the mean \pm SD of four independent experiments.
$153 \mathrm{bp}$ was found in B16-F10 cells transfected with pcDNA3.1/gef for different time periods, indicating the effectiveness and ability of the construction in order to be used in the subsequent experiment. To demonstrate the integrity of the RNA preparations, PCR was performed using $\beta$-actin primers. Analysis of the bands, normalized by comparison with the $\beta$-actin signal, showed a progressive increase of gef expression; this was 3.9- and 4.5-fold higher at 48 and $72 \mathrm{~h}$ vs B16-F10 cells at $24 \mathrm{~h}$ and was maximal at $96 \mathrm{~h}$ after transfection (sixfold higher vs B16-F10 cells at $24 \mathrm{~h}$ ). Previously, cell transfection was optimized by pcDNA3.1/GFP (Fig. 1b). As shown in Fig. 1c, the B16-F10 cells transfected with pcDNA3.1/gef showed a significant and time-dependent decrease in growth. Twenty-four hours after transfection a $28 \%$ decrease in growth rate versus control cultures was observed. The decrease in proliferation was $45.5 \%$ at $48 \mathrm{~h}$. The main decrease in proliferation rate occurred at $72 \mathrm{~h}$ and $96 \mathrm{~h}$, when similar ratios of growth decrease were observed $(64.6 \%$ and $69.7 \%$, respectively). In contrast, the growth of B16-F10 cells transfected with the empty vector (control group) was similar to that of the parental cells.

\section{Expression of gef gene-induced apoptosis in B16-F10 cells}

Apoptotic rates of B16-F10 cells untreated or transfected with empty vector revealed no significant difference. Only $8.7 \%$ of the pcDNA3.1/gef transfected cells showed apoptosis after $24 \mathrm{~h}$. However, at 48 and $72 \mathrm{~h}$ a significant increase was found (19\% and 34\%, respectively) (Fig. 2a). At $96 \mathrm{~h}$ apoptosis was similar to that found at $72 \mathrm{~h}$ (data not shown). These results indicated the ability of gef gene to stimulate apoptosis in B16-F10 melanoma cells after in vitro transfection. The induction of apoptosis by gef gene was also evident by confocal laser-scanning microscopy (Fig. 2b). Furthermore, to confirm whether the growth inhibitory effects of gef gene are related to the induction of apoptosis, we used an ELISA-based assay. Amounts of cytoplasmic oligonucleosomes (an indicator of apoptosis) increased between 24 and $96 \mathrm{~h}$ after gef transfection as compared with untreated cells. As shown in Fig. 2c, the strongest enrichment factor was obtained at 72 and $96 \mathrm{~h}$ (3.6 and 5.4, respectively). These results provide convincing data that up-regulation of gef induces apoptosis in B16-F10 cancer cells.

\section{Gef gene therapy-induced modulation of mitochondrial membrane potential and caspase- 9 activation}

To determine if induced apoptosis by gef gene in B16-F10 cells is mediated via the mitochondrial pathway, mitochondrial membrane integrity was measured by DiOC6(3) dye staining. As shown in Fig. 3a, a significant decrease in 

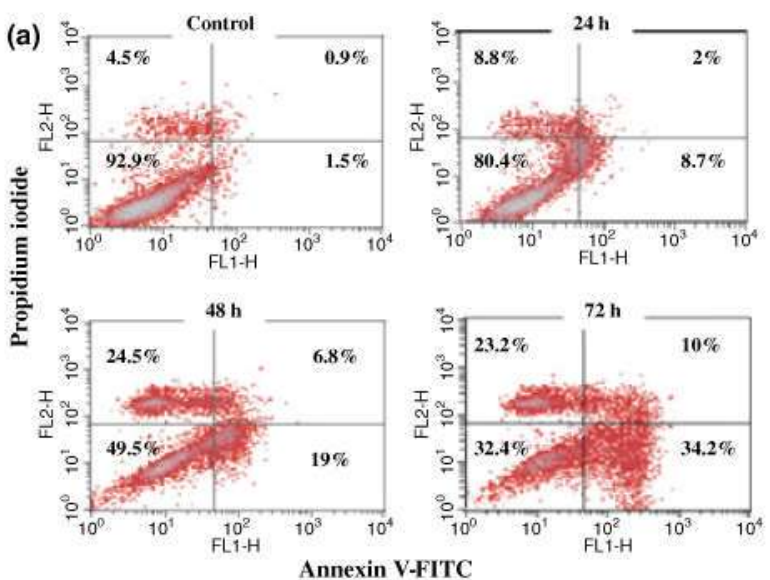

(b)
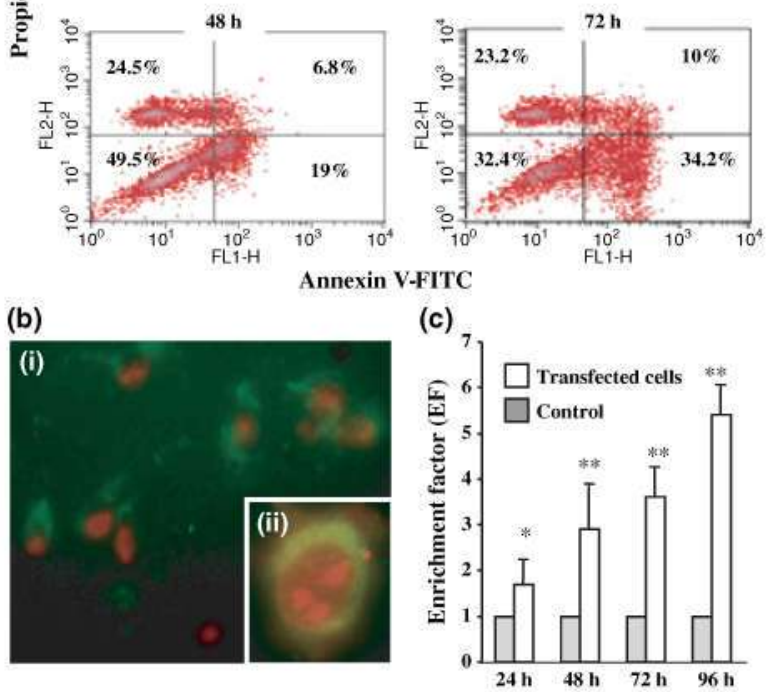

Figure 2. Analysis of apoptosis induction by gef gene in B16-F10 cells. (a) Fluorescence-activated cell sorting. Cells were stained with annexin $V$ and propidium iodide (PI) to evaluate apoptotic cell death, as described in Methods. These data are representative results from four separate experiments. (b) Annexin V-FITC staining and confocal microscopy. The annexin V-FITC fluorescence localized at the periphery of the cells consequent to the translocation of phosphatidylserine (PS) residues from the inner leaflet of the plasma membrane to the outer leaflet. B16-F10 cells $48 \mathrm{~h}$ after transfection (a). Cell nuclei were counterstained with PI before examination under a confocal laserscanning microscope. A stronger binding was observed when the study was carried out $96 \mathrm{~h}$ after gef transfection (b). The experiment was performed three times with identical results. Magnification: (a) 20x; (b) $40 \times$. (c) ELISA apoptosis assay of cytoplasmic nucleosomes. The specific enrichment of mono- and oligonucleosomes released into the cytoplasm was calculated using the formula: mean of absorbance of transfected cells/mean of absorbance of control cells = enrichment factor (EF). The EF was calculated relative to the control value $(=1)$ of untreated cells. ${ }^{*} P<0.05 ; * * P<0.01$ compared to control cells.

membrane potential was detected in transfected cells at 24 , 48 and $72 \mathrm{~h}$ compared with parental cells (control) indicating a mitochondrial membrane permeability increase after gef gene treatment. At $96 \mathrm{~h}$ membrane potential modulation was similar to that found at $72 \mathrm{~h}$ (data not shown). Caspase-9 activity was induced after gef gene treatment in B16-F10 cells. Although its activity was modulated at different times, the largest increase was observed at 72 and $96 \mathrm{~h}$ (three- and 4.1-fold; respectively). The caspase- 8 activity remained unchanged (Fig. 3b). These data support the hypothesis that gef gene-induced apoptosis through the mitochondrial-mediated pathway. (a)
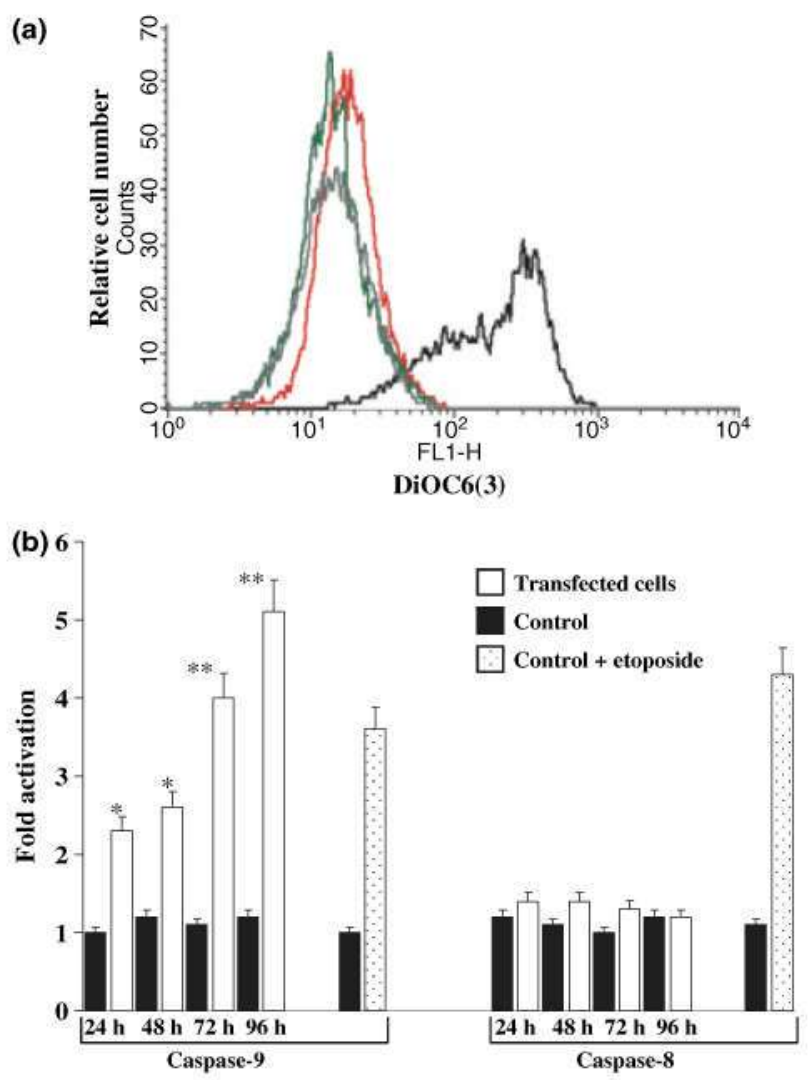

Figure 3. Mitochondrial membrane potential and caspase activity. (a) Reduction of mitochondrial transmembrane potential $\left(\Delta \psi_{\mathrm{m}}\right)$ in B16F10 cells (black) after $24 \mathrm{~h}$ (red), $48 \mathrm{~h}$ (grey) and $72 \mathrm{~h}$ (green) of gef transfection. DiOC6(3) was added to the culture medium during the last $15 \mathrm{~min}$ of treatment at a final concentration of $40 \mathrm{~nm}$. The fluorescence intensity of DiOC6(3) was analysed by flow cytometry. Data shown are representative of three independent experiments. (b) Caspase-9 and -8 activities were determined (as described in Methods) in pcDNA 3.1/gef transfected B16-F10 cells at indicated time points in comparison with parental cells (percentage values). Etoposide treatment ( 6 h) was used to demonstrate caspase 8 and caspase 9 activities in B16-F10 cells. Experiments were performed four times with identical results. ${ }^{\star} P<0.05 ; * * P<0.01$ compared to control cells.

\section{Gef gene effects on melanoma growth in vivo}

The potential of gef gene to promote tumor cell killing in vivo was evaluated by direct injection of the plasmid complexed with jetPEI in B16-F10 subcutaneous mice tumors. Figure 4 shows that gef gene was able to inhibit tumor growth. During the first 2 days following treatment, tumor volume decreased by $40.4 \%$ in the gef gene treated group, as compared with the control group. On post-treatment days 4 and 6 , the observed reduction was $45.2 \%$ and $54.3 \%$ respectively. Following a similar trend, a $77.7 \%$ volume reduction was observed on day 8 . After this time, the control group showed a high mortality rate (Fig. 4). Mice treated with gef gene showed no evidence of systemic toxicity (i.e. animal death, loss of body weight, other tissue damage 


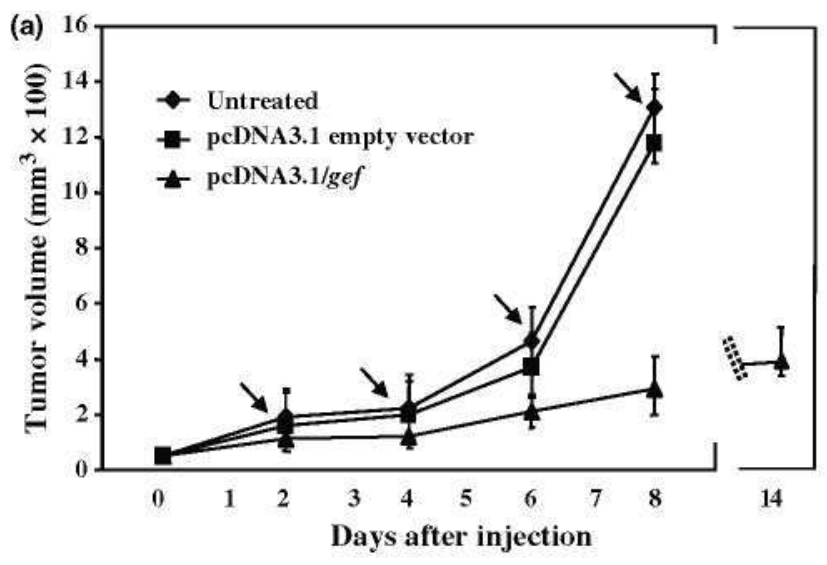

(b)

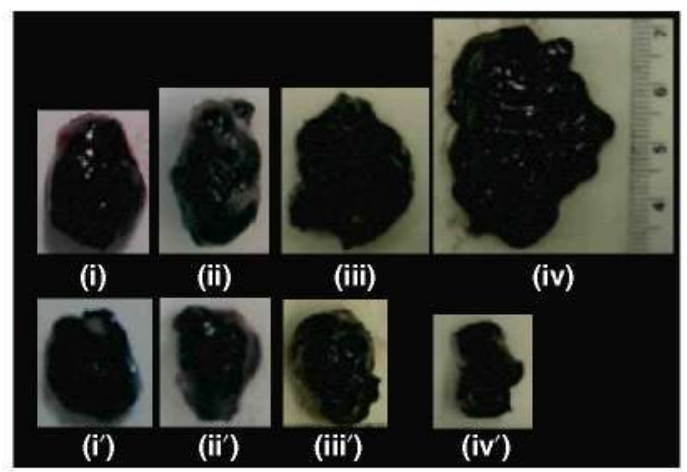

(c)

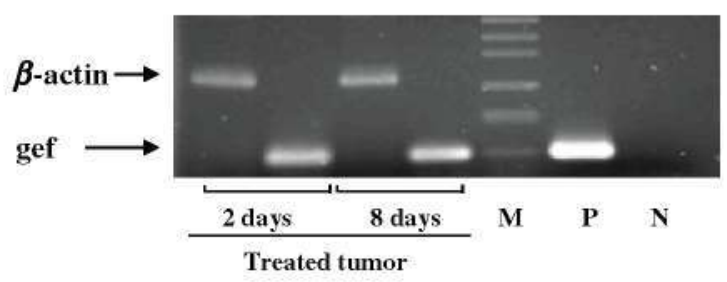

Figure 4. Effect of direct intra-tumoral injection of the gef gene on the growth of subcutaneous tumor induced by B16-F10 tumors cells in mouse. (a) Tumor volume variation after gef gene treatment. Time of injection is indicated through arrows; treatment began (day 0 ) when tumors had reached a volume of $75 \mathrm{~mm}^{3}$. The plot shows a significant tumor volume reduction in the treated group $(n=14)$ as compared with the control groups, transfected with empty vector $(n=7)$ and without treatment $(n=7)$. (b) Representative gross appearance of tumors excised from mice sacrificed during treatment at $2\left(a^{\prime}\right), 4\left(b^{\prime}\right) 6$ $\left(c^{\prime}\right)$ and $8\left(d^{\prime}\right)$ days and tumors obtained from mice without treatment at the same time intervals ( $a, b, c$ and $d$, respectively). (c) Determination of gef gene expression in tumor mice after 2 and 8 days of pCDNA3.1/gef treatment. The integrity of the RNA tissue used was determined using $\beta$-actin primers. $M$, molecular weight; $P$, positive control (pcDNA3.1/gef); $N$, negative control (mouse tumors without treatment).

or changes in behaviour or aspect). After empty vector injection with jetPEI, no tumor growth modifications were observed (Fig. 4a,b). RT-PCR was performed to ensure gef gene expression in all treated tumors. Figure $4 \mathrm{c}$ shows a comparison of gef gene expression in melanoma tissue between days 2 and 8 after the treatment.

\section{gef gene-induced apoptosis in melanoma cells in vivo}

To determine the gef expression efficiency to induce apoptosis in vivo we analysed established subcutaneous B16-F10 tumors with a TUNEL reaction mixture. As showed in Fig. 5a, the number of apoptotic cells (green) was significantly higher in tumors treated with pcDNA3.1/gef in comparison with the tissue control. Analysis of the melanoma sections showed a progressive increase of per cent apoptosis which was maximal at 8 days after treatment (Fig. 5b). To examine possible caspase- 9 or -8 -activation by gef gene treatment we used immunohistochemistry. Weak or absent expression of caspase- 8 protein was detected in tissue samples (data not shown). However, all samples of melanoma tissue treated with pcDNA3.1/gef at different times showed a clear caspase-9 activation, with the strongest staining observed from the 4th day of treatment until the end of the experiment. Caspase- 9 expression was not seen in any of the controls or in melanoma tissue treated with the empty vector (Fig. 5c).

\section{Transmission electron microscopy}

To further investigate the nature of gef gene-mediated cytotoxicity, B16-F10 mouse melanoma-induced tumors treated with gef gene in vivo were analysed by TEM. The control tissue showed giant malignant cells with an intact cell membrane and single- or multiple-nuclei cells. The first identifiable morphological change after treatment was the pronounced swelling in the mitochondria seen within 2 days of treatment. Mitochondria in control cells and cells treated with vehicle alone remained unaffected. Forty-eight hours later, apoptosis ultrastructural characteristics, such as chromatin condensation, crescent formation and margination were seen by electron microscopy in the treated melanoma, but not in the control group. Similar pictures were observed 6 and 8 days after treatment (Fig. 6).

\section{Discussion}

New treatment strategies for malignant melanoma are urgently needed because conventional approaches like chemotherapy and radiation have little impact on patient survival in the advanced stages of the disease. As a promising alternative, gene therapeutic strategies based on suicide gene expression in tumor cells have been developed. In this study, we have demonstrated the in vivo potential use of the gef cDNA as a suicide gene in a new melanoma gene therapy approach.

To date, classical suicide gene therapy systems have not guaranteed the successful treatment of melanoma and have 


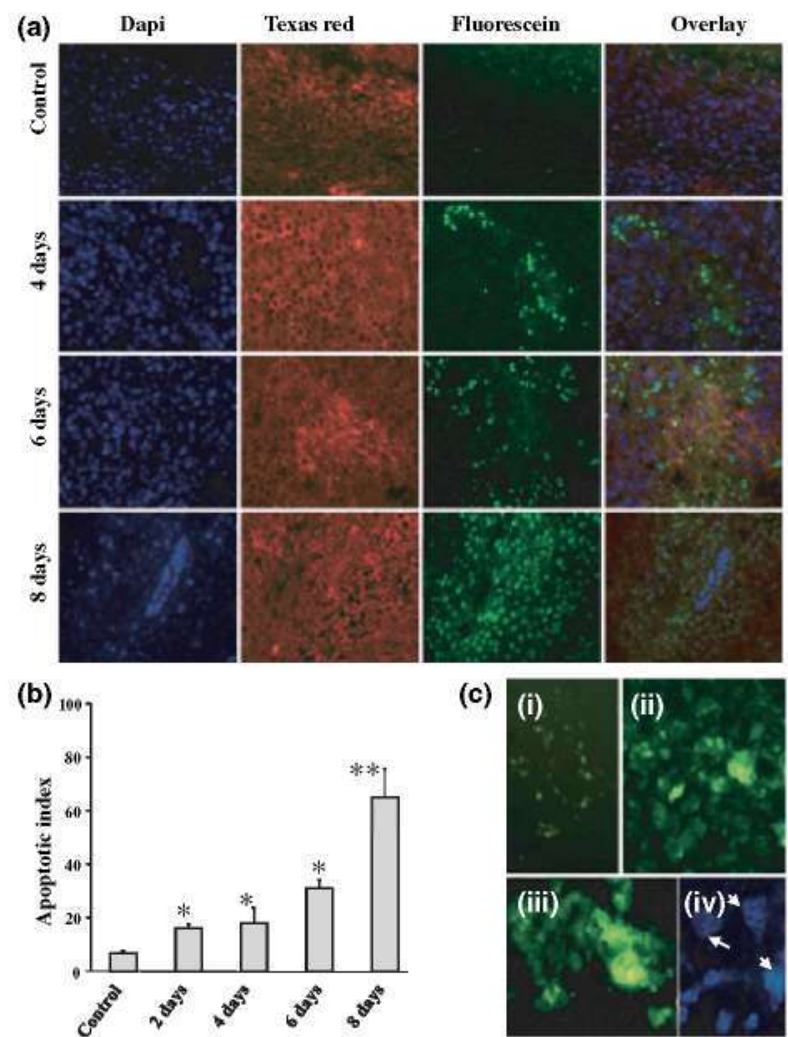

Figure 5. Histological evaluation of apoptosis and caspase activity of melanoma cells in vivo. (a) Representative photographs of tumor sections showing TUNEL-positive cells (green). Sections were counterstained with DAPI (blue) and anti-tubulin (red). Apoptotic cells increased in tumors treated with pcDNA 3.1/gef at 2 (data not shown), 4,6 and 8 days compared to untreated tumors (control) $(\times 20)$. (b) Per cent apoptosis in each group. Values were expressed as mean \pm SD. Level of significance compared to control cells: ${ }^{*} P<0.05 ; * * P<0.01$. (c) Caspase expression in melanoma tumor was detected using immunofluorescence staining. Microscopic analysis showed that melanoma tissue after treatment with pcDNA3.1/gef was strongly caspase-9-positive. Untreated tumors (a) and melanoma tumor after 4 (b) $(40 x)$ and 6 (c) $(60 x)$ days of treatment. Cell nuclei counterstained with DAPI showed that some cells displayed apoptotic morphology and nuclear segmentation (arrows) (d, 60x). All data were obtained from the study of at least three tumors.

induced only a partially positive response $(16,17)$. One of the main limitations of these indirect action systems is the need to use prodrugs (18).The use of genes encoding toxins avoids the administration of a prodrug, eliminating its side-effects, its bioavailability limitations and the consecutive applications of vector and prodrug. Moreover, these genes can be directly expressed in the cytosol of the target cells, thus overcoming the problems (cytotoxicity, internalization efficiency and resistance acquired by cancer cells) originated by their use as components of immunotoxins or recombinant chimeras (19). In melanomas, viral genes encoding toxins such as viral protein $\mathrm{R}$ and some plant
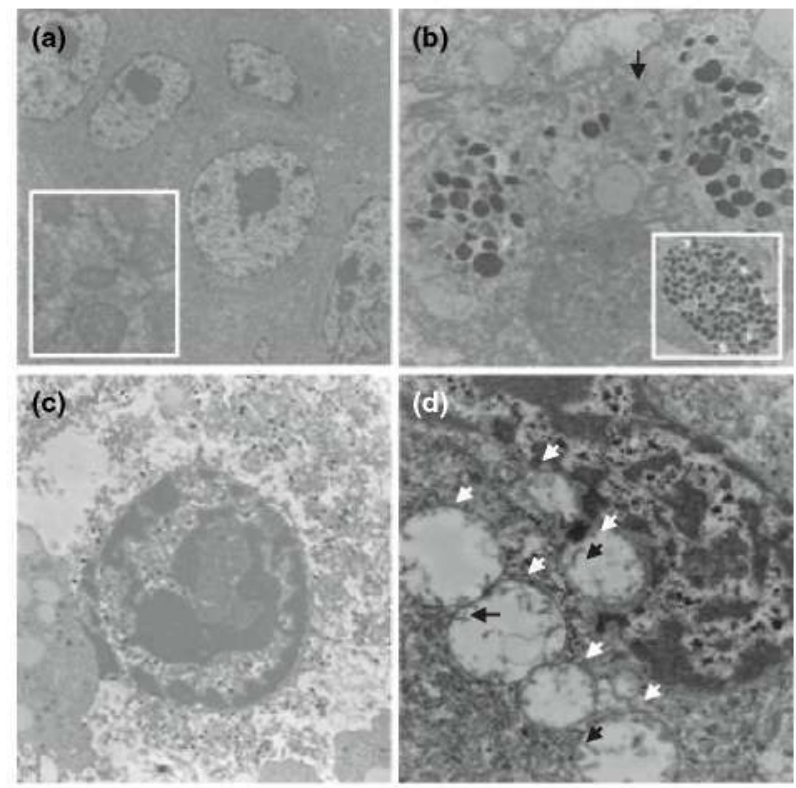

Figure 6. Transmission electron microscopy of melanoma tumors without treatment showed typical tumor cells with polygonal shape, large nucleus, light cytoplasmic complexion containing well preserved organelles (a) including mitochondrias ( $A$ insert) (1100x) and a large amount melanosomes (B insert) including premelanosomes (arrows) (b) $(\times 4000)$. Representative photomicrograph of melanoma treated with pCDNA3.1/gef ( 6 days) showing ultrastructural characteristics of apoptosis such as chromatin condensation, crescent formation and margination (c) ( $\times 6300)$. Note, the nucleus near of the swollen mitochondrias (white arrows) with disrupted cristae (black arrows) (d) (12000x). Data were obtained from the study of at least three tumors.

genes such as saporin (SAP) have been applied with a significant result to induce tumoral cell death $(20,21)$. Our previous results in vitro showed that gef is effective in melanoma MS-36TG cells, modulating their proliferation capacity, differentiation degree and tumor malignancy (22). In the present study, we have demonstrated that the transfection of the pcDNA $3.1 / \mathrm{gef}$ not only inhibits in vitro melanoma proliferation but also it is highly toxic for tumors in vivo. The gef gene treatment induced a significant decrease in tumor growth $(77.7 \%$ relative volume reduction after 8 days of treatment), an effect that was clearly improved upon repeated administrations. Experimental treatment with HSV-tk/GCV (23) or more recently with the SAP gene (21) in the same tumor type, induced a $40-50 \%$ and $67 \%$ relative volume reduction, respectively. McCray et al. (20), who used the Vpr gene integrated in the pcDNA3.1 $(100 \mu \mathrm{g})$ vector in melanoma tumor from B16-F10 culture cells, described an $86 \%$ of tumor volume reduction which required 25 days of treatment. Therefore, the main advantage of the gef gene is not only its efficacy in melanoma cells but also the shorter latency for effective antitumoral action. 
Although we have demonstrated the cylotoxic effect of gef gene in cancer cells, the specific mechanism of action has remained unclear so far. In prokaryotic cells, the gef gene diminishes the membrane potential, leads to membrane leakiness and also induces morphological changes (24). Eukaryotic cells fundamentally differ from prokaryotic cells in terms of their cellular structure, organization, metabolism and membrane composition. Nevertheless, as the eukaryotic endomembrane system arose in an ancestral prokaryotic lineage (25) gef gene might act in cell organelle membranes. Recently, it was demonstrated that breast cancer cells growth was inhibited by bacteriophage $\lambda$-holin, a protein that can permeabilize the bacterial membrane (12). Our results showed that $48 \mathrm{~h}$ after induction B16-F10 cells become multinucleated, in some cases extensively vacuolated and finally detached from the culture dish surface. Experiments with annexin, confocal laser-scanning microscopy and nucleosomes clearly showed that the gef gene is able to induce apoptosis in a timedependent manner. These results are similar to those obtained with the SAP gene which also induces programmed cell death and direct DNA fragmentation in B16-F10 cells (21). Interestingly, the pronounced clinical chemoresistance of melanoma is strongly suggestive of an inactivation of apoptotic programmes. Defects in proapoptotic signalling pathways and enhancement of antiapoptotic pathways may synergistically contribute to this apoptosis deficiency (26). Immunohistochemical analysis by TUNEL assay revealed that $\mathrm{pcDNA3.1/gef}$ treatment significantly increased apoptosis in established subcutaneous B16-F10 tumors in vivo. The incidence of apoptosis in the tumor almost corresponded to the effect of tumor growth inhibition, suggesting that our experimental treatment resulted in tumor regression by significant augmentation of apoptosis.

Apoptosis may occur via death-receptor dependent (extrinsic) or mitochondrial (intrinsic) pathways. The extrinsic pathway is triggered by the activation of death receptors, such as Fas and TRAIL receptors (DR4, DR5) activating initiator caspase-8, which then cleaves executioner caspase-3. The mitochondrial pathway of cell death is mediated by $\mathrm{Bcl}-2$ family proteins, which disrupt the mitochondria membrane potential and result in release of apoptogenic factors, such as cytochrome $\mathfrak{c}$, from the mitochondria into cytosol; in turn, these factors would form an apoptosome with apoptosis activating factor 1 and caspase-9 (27). Treatments modulating apoptosis phenomenon, for example with bcl-2-targeted antisense, are a promising new strategy in melanoma (28). Assays with drugs such as hydroquinone or thiobenzanilides in this tumor type have demonstrated an action mechanism related to caspase- 9 activation $(29,30)$. This tumoral cellular injury mediated by caspases may also be induced by suicide genes and it may be relevant in relation to their application in tumors. In fact, $\mathrm{CD} / 5 \mathrm{FC}$ system induces apoptosis in human malignant glioma cells by the activation of caspases- 3 and -9 but not caspase-8 (31) while a certain modification, the bifunctional $E$. coli cytosine deaminase and uracil phosphoribosyltransferasefusion, is able to induce caspase- 3 activation only (32). HSV $t k / \mathrm{GCV}$ activates caspase- $3,-8$ and -9 in rat bladder carcinomas (33) and a variant, the thymidylate kinase, induces apoptosis in Jurkat cells by activation of caspase-3 only (34). Our studies in B16-F10 cells expressing gef showed alteration of the mitochondrial membrane integrity suggesting that apoptosis is mediated by the mitochondrial pathway. This hypothesis is supported by the caspase- 9 activity increase in B16-F10 transfected cells. Moreover, the mitochondrial transmembrane potential is altered in most of the cellular population, supporting the hypothesis on the possible effect of gef once it is released from the apoptotic cells. The mitochondrial-mediated apoptotic pathway is strongly supported by our ultrastructural findings in the induced B16-F10 tumors in mice which showed dilated mitochondria with disrupted cristae. Moreover, the in vivo assay shows that caspase- 9 activity increases significantly after gef gene treatment, supporting the participation of a mitochondrial-mediated apoptotic pathway in our gene therapy system. However, we can not exclude the possible participation of other apoptosis-mediated molecule in treated B16-Fl0-induced tumors such as endonuclease G, Smac/DIABLO and HtrA2 (35). Further studies are required to elucidate the exact mechanisms involved.

We have reported the successful use of the gef gene as an anticancer gene therapy system, not only in melanoma cells in culture but in melanoma tumors in vivo. Our in vivo experiments show that gef gene has a rapid and efficient activity in relation to tumor volume decrease. However, gef gene binds to the mitochondrial membrane and its activity is not tumor-specific. Therefore, it will be necessary to create this specificity as in most of the toxic genes used in gene therapy (8-12). We have injected intra-tumorally the pcDNA3.1/gef plasmid to observe its activity in melanoma cells. Adenoviral vectors modified by attaching tumorspecific promoters should be used to assay metastatic melanoma treatment. Currently, we are using specific enhancer/promoter genes (such as tyrosinase) (36), new vectors (such as ReCon) (37) and combined therapy with cytotoxic drugs (38) to improve the tumoral response against gef gene. Moreover, it will be necessary to demonstrate the apoptosis induction in human melanoma by the extopic gef gene expression. In summary, our results suggest that gef is a suicide gene candidate for oncologic in vivo applications and that it may contribute to eradicate tumor mass in combination with surgery or classic radioor chemotherapy. 


\section{Acknowledgements}

We thank G. Oriz Ferrón for excellent technical assistance and for providing the poDNA3.1/GFP plasmid. We are also thankfil to Dr F. O'Valle (Department of Anatomopathology, Granada University) for her help and suggestions and Dr M. Burgos (Department of Genetics, Granada University) for plasmid constructs. This study was supported by the Fondo Investigaciones Sanitarias Seguridad Social (FIS), Project no. Pl041372 and by Granada University Project no. UGR-30 B364 1101/2007.

\section{References}

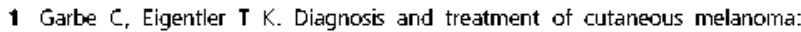
state of the art Melanoma Res 2007: 17 117-127.

2 Katipamula R, Markovic $5 \mathrm{~N}$. Emerging therapies for melanoma Exper Rev Anticancer Ther 2008: 8: 553-560.

3 Eberle J, Fexker L F, Hossini A $M$ et at. Apoptosis pathways and oncolytic adenoviral vectors: promising targets and tools to overcome therapy resistance of malignant melanoma. Exp Dermatol 2008: 17 1-11

4 Boehm I. Apoptosis in physiological and pathological skin: implications for therapy. Curr Mol Med 2006: 6: 375-394.

5 Altaner C. Prodrug cancer gene therapy. Cancer lat 2008: 270: 191-201.

6 Slade N, Galetić I, Kapitanović S, Pavelić J. The efficacy of retroviral herpes simplex virus thymidine kinase gene transfer and ganciclovir treatment on the inhibition of melanoma growth in vitro and in vivo. Arch Dermatol Res 2005 293. $484-490$

7 Liu $Y$, Deisseroth $A$. Oncolytic adenoviral vector carrying the cytosine deaminase gene for melanoma gene therapy Cancer Gene Ther 2006: 13: 845-855

8 Zitzer $\mathrm{A}$, Palmer $\mathrm{M}$, Weller $\mathrm{U}$ et at. Mode of primary binding to target membranes and pore formation induced by Vibrio cholerae cylolysin (hemolysin). Eur J Biochem 1997: 247 209-216.

9 Frankel AE, Powell B L, Duesbery N S et af. Anthrax fusion protein therapy of cancer Curr Protein Pept Sci 2002- 3: 399-407.

10 Rustamzadeh E, Hall W A, Todhunter D A et af. Intracranial therapy of glioblastoma with the fusion protein DTAT in immunodeficient mice. Int I Cancer 2007: 120-411-419

11 Geden S E. Gardner R A. Fabbrini M S et af. Lipopolyamine treatment increases the efficacy of intoxication with saporin and an anticancer saporin conjugate. FEBS J 2007: 274: 4825-4836.

12 Agu C A. Klein R. Schwab S et at. The cytotoxic activity of the bacteriophage lamb-da-holin protein reduces tumour growth rates in mammary cancer cell xenograft models. J Gene Med 2006: 8: 229-241

13 Poulsen L K, Rein A, Molin S. Andersson P. Topographic analysis of the toxic gef protein from E.colf. Mol Microbiol 1991-5. 1639-1648.

14 Boulaiz $\mathrm{H}$, Prados J, Melguizo $C$ et at. Inhibition of growth and induction of apoptosis induction in human breast by transfection of gef gene. $\mathrm{Br} J$ Cance 2003: 69 : 192-198.

15 Boulaiz H, Prados J, Marchal J A et af. Transfection of MS-36 melanoma cells with gef gene inhibits proliferation and induces modulation of cell cycle. Cancer Sci 2003: 94: 564-568.

16 Sanchez-Perez $L$, Gough $M$. Qiao J et at. Synergy of adoptive T-cell therapy and intratumoral suicide gene therapy is mediated by host NK cells. Gene Ther 2007: 14: 998-1009.

17 Zamboni S, Mallano A, Flego M et af. Genetic construction, expression, and characterization of a single chain anti-CEA antibody fused to cytosine deaminase from yeast. Int J Oncol 2008-32-1245-1251.
18 McKeown $S R$, Ward $C$, Robson $T$. Gene-directed enzyme prodrug therapy: a current assessment. Curr Opin Mol Ther 2004: 6: 421-435.

19 Frankel A, Kreitman R, Sausville E. Targeted toxins Clin Cancer Res 2000- 6 $326-334$.

20 McCray A $N$, Ugen $K E$, Muthumani $K$ et at. Complete regression of established subcutaneous B16 murine melanoma tumors after delivery of an HV-1 $\checkmark p r$-expressing plasmid by in wivo electroporation. Mol Ther 2006: 14: 647655.

21 Zarowni $N$, Vago $R$, Soldá $T$ et at. Saporin as a novel suicide gene in anticancer gene therapy. Cancer Gene Ther 2007: 14: 165-173.

22 Boulaiz $\mathrm{H}_{r}$ Prados $J$, Melguizo $\mathrm{C}$ et at. Tumour malignancy loss and cell differentiation are associated with induction of gef gene in human melanoma cells Br J Dermatol 2008: 159: 370-378.

23 Soubrane $C$, Mouawad R, Rixe $O$ et af. Direct gene transfer of a plasmid carrying the herpes simplex virus-thymidine kinase gene (HSV-TK) in transplanted murine melanoma in vivo study. Eur J Cancer 1996: 32. 691-695.

24 Ronchel $M$, Ramos J L Dual system to reinforce biological containment of recombinant bacteria designed for rhizomediation. Appl Enwiron Microbiol 2001: 67: 2649-2656.

25 Emelyanov $\vee V$. Mitochondrial connection to the origin of the eukaryotic cell. Eur J Biochem 2003: 270-1599-1618.

26 Soengas M S, Lowe S W. Apoptosis and melanoma chemoresistance. Oncogene 2003: 22: $3138-3151$

$27 \mathrm{Kim} R$ Recent advances in understanding the cell death pathways activated by anticancer therapy. Cancer 2005: 103-1551-1560.

28 Moreira I N, Santos A. Simós S. BCl-2-targeted antisense therapy (Oblimersen sodium): towards clinical reality. Rev Recent Clin Trias 2006: 1: 217235

29 Fernandes $N$, Jung $M$, Daoud $A$, Mo $H$. Biphenylalkylacetylhydroquinone ethers suppress the proliferation of murine B16 melanoma cells Anticancer Res 2008: 28: 1005-1012

$30 \mathrm{Hu} W \mathrm{P}, \mathrm{YU} \mathbf{H} \mathrm{S}$, Chen $\mathrm{Y} R$ et at. Synthesis and biological evaluation of thiobenzanilides as anticancer agents. Bloorg Med Chem 2008- 16-52955302.

31 Kurozumi K. Tamiya $T$. Ono $Y$ et af. Apoptosis induction with 5-fluorocytosine/cytosine deaminase gene therapy for human malignant glioma cells medlated by adenovirus. J Neurooncol 2004: 66: 117-127

32 Gopinath $\mathrm{P}$, Ghosh S S. Apoptotic induction with bifunctional $E$. cof cytosine deaminase-uracil phosphoribosyltransferase mediated suicide gene therapy is synergized by curcumin treatment in vitro. Mol Biotechnol 2007 . 39: $39-48$.

33 Shibata M A, Horiguchi T. Morimoto J, Otsuki Y Massive apoptotic cell death in chemically induced rat urinary bladder carcinomas following in situ HSVtk elertrogene transier. J Gene Med 2003 5 : 219-231.

34 Sato T, Neschadim A, Konrad M, Fowler D H, Lavie A, Medin J A. Engineered human tmpk/AZT as a novel enzyme/prodrug axis for suicide gene therapy Mol Ther 2007- 15: 962-970

35 Keeble J A, Gilmore A. P. Apoptosis commitment - translating survival signals into decisions on mitochondria Cell Res $2007 \cdot 17 \cdot 976-984$

36 Fecker L F, Geilen C C. Hossini A M et al. Selective induction of apoptosis in melanoma cells by tyrosinase promoter-controlled CD95 ligand overexpression I Invert Dermatol 2005: 124: 221-228.

37 Brandtner E M, Kodajova P, Hlavaty J et at Reconstituting retroviral (ReCon) vectors facilitating delivery of cytotoxic gen in cancer gene therapy approaches. J Gene Med 2008-10: 113-122

38 Prados J. Melguizo C. Rama A et at. Combined therapy using suicide gef gene and paclitaxel enhances growh inhibition of multicellular tumour spheroids ot A-549 human lung cancer cells. Int J Oncol 2008-33-121-127. 